\title{
Planar Time Optimal Paths for Non-Symmetric Vehicles in Constant Flows
}

\author{
Bilal Hammoud and Elie Shammas \\ Department of Mechanical Engineering \\ American University of Beirut \\ Beirut, Lebanon
}

\begin{abstract}
This paper introduces a new kinematic model to describe the planar motion of an Autonomous Underwater Vehicle (AUV) moving in constant current flows. The AUV is modeled as a rigid body moving at maximum attainable forward velocity with symmetric bounds on the control input for the turning rate. The model incorporates the effect a flow will induce on the turning rate of the AUV due to the non-symmetric geometry of the vehicle. The model is then used to characterize and construct the minimum time paths that take the AUV from a given initial configuration to a final configuration in the plane. Two algorithms for the time-optimal path synthesis problem are also introduced along with several simulations to validate the proposed method.
\end{abstract}

\section{INTRODUCTION}

The path planning problem is a fundamental problem in the field of autonomous robotics. In the late 1880's Markov [15] introduced the following minimum time problem: given any initial and final positions and headings in a plane, what is the minimum length trajectory, given that there exists an upper and lower bound on the curvature of the curve. In 1957, L. E. Dubins [12] presented his solution to the Markov problem showing that a solution exists and comprises of two arcs of maximum turning rate joined by a straight line, or three maximum turning rate arcs if the Euclidean distance between the initial and final positions is less than four minimum turning radii. While Dubins solved the problem using geometric arguments only, Boissonnat et al. [5] solved the minimum time problem using optimal control theory. The complete path synthesis problem for the Dubins car model was solved by Bui et al. [6].

Many variations of the Dubins-Markov problem were presented later on. Reeds and Shepp [19] solved the minimum time problem for a Dubins car that goes both forwards and backwards, thereby introducing cusps to the solution presented by Dubins. With most of the minimum time path planning work being done on car like robots, Balkcom and Mason [3] presented the minimum time paths for a differential drive robot. They identified 40 different classes of candidate optimal trajectories and developed an algorithm to solve for the time optimal path for a differential drive robot. Balkcom et al. [4] also investigated the minimum time problem for an omniwheeled robot. Furtuna and Balkcom [13] described the structure of the optimal trajectories for symmetric systems such as the dubins car, the differential drive and omnidirectional systems.
Chitsaz and LaValle [8] introduced the Dubins airplane model extending the Dubins car model into $3 D$ space and characterized the corresponding candidate time optimal trajectories. McGee and Hedrick [16] and Bakolas and Tsiotras [1] characterized and solved for the minimum time paths of a planar Dubins airplane model traveling in constant winds. More recently, McNeely et al. [17] proved the existence and uniqueness of minimum time trajectories for a Dubins vehicle flying in time varying winds, and then introduced an algorithm for obtaining the minimum time paths for such vehicles.

Bakolas and Tsiotras [2] considered a Dubins car model having non symmetric bounds on the control input. The structure of the optimal control is the same as that of the Dubins model but the synthesis problem turned out to be significantly different. Choi [9] studied Dubins vehicles experiencing severe damages or control failure. In the presented cases a unidirectional constraint is specified and the lower and upper bounds on the turn rate take the same sign, meaning that the vehicle is always rotating to one side at a turning rate $\in[a, b]$ where $a$ and $b$ are of the same sign. Choi then solved the minimum time path planning problem and extended the planar results to the planning of aircraft emergency landing.

Rysdyk [20] explained that an airplane moving in a constant flow field and executing maximum turn rates would traverse a trochoidal path. Techy and Woolsey [22] used Rysdyk's results to obtain analytical solutions for the minimum time path planning problem of a planar airplane in a constant flow field. A more recent work was published by Dolinskaya et al. [11] where the direction dependence of the minimum turning radius and the maximum forward velocity of a ship traveling in sea currents are considered. Chang et al. [7] developed similar work, where the minimum turning radius of robots working in mines depends on the slope and nature of the ground making it direction-dependent. In both cases, the minimum time path planning was addressed, and a general characterization of the optimal path structures for general direction-dependent models was discussed.

Most of the work done previously considered the agent as a particle moving either in a $2 D$ plane or in a $3 D$ space with a heading angle defined. Considering the system as a particle ignores any interaction between the system and the surrounding environment other than translational effects due to surrounding winds or flows. This paper introduces a novel kinematic model that captures the rotational effects due to 
constant flows interacting with a non-symmetric geometry of a body. A minimum time solution for the path planning problem is then presented through the use of optimal control theory and geometric arguments.

The paper is organized as follows, the model is introduced and analyzed in Section III. Then using Pontryagin's Minimum Principle [18], the space of feasible solutions is reduced to path concatenations of straight segments and arcs of maximum turn rates in Section III. In Section IV, the structure of the paths under the optimal motion primitives is characterized. After that, using geometric arguments and results from previous work done by Dolinskaya and Maggiar [10] and Sussmann and Tang [21] the space of solutions is further reduced to concatenations consisting of a maximum of five segments. Imposing further restrictions on the flow velocity, the solution is reduced into concatenations of three segments, and a corresponding path planning solution is proposed in Section V. Finally, several simulations depicting the proposed algorithms are shown in Section VI

\section{Motion Models}

The first planar motion model introduced was the Dubins car model described in (1), which was later modified by McGee and Hedrick [16] to describe an airplane traveling in constant winds described in (2). The model proposed in this paper is inspired by the previously mentioned models.

In the Dubins car model [12], the system is modeled as a particle with a defined direction in a $2 \mathrm{D}$ plane. The optimal path planning problem is to find the minimum time trajectory from an initial configuration to a final configuration. Let $q=$ $(x, y, \theta)$ be an element of the configuration space $Q=\mathbb{R}^{2} \times \mathbb{S}$. The kinematic model used in the formulation of the problem is presented in (1), where the particle is assumed to be moving at a constant forward velocity, $v$, which is typically equal to unity.

$$
\begin{aligned}
\dot{x}(t) & =v \cos \theta(t), \\
\dot{y}(t) & =v \sin \theta(t), \\
\dot{\theta}(t) & =u(t),
\end{aligned}
$$

where $u(t) \in\left[-u_{\max }, u_{\text {max }}\right]$ is the control input on the rotation speed with $u_{\max }=1$. Another model proposed by McGee and Hedrick [16] in (2) introduces the translational effects a constant flow will induce on the particle.

$$
\begin{aligned}
\dot{x}(t) & =v \cos \theta(t)+\eta \cos \phi, \\
\dot{y}(t) & =v \sin \theta(t)+\eta \sin \phi, \\
\dot{\theta}(t) & =u(t),
\end{aligned}
$$

where $\eta$ and $\phi$ are the speed and direction of the constant flow, respectively. Model (2), which shall be labeled as the irrotational model, was extensively used in the literature for solving the minimum time path problem for unmanned aerial vehicles and underwater autonomous vehicles. However, this model only accounts for the translational effects of the flow while it ignores any other interactions between the vehicle and the flow, namely, the rotational effects.

In order to capture more details of how an interaction might cause the vehicle to rotate, an additional term is added to the third equation in (2) to arrive at the novel model given in (3). This proposed model, labeled as the rotational model, assumes that the effect on rate of change of the heading angle is proportional to the perpendicular component of the flow velocity with respect to the heading angle of the vehicle as shown in Fig. 1 .

$$
\begin{aligned}
\dot{x}(t) & =v \cos \theta(t)+\eta \cos \phi \\
\dot{y}(t) & =v \sin \theta(t)+\eta \sin \phi \\
\dot{\theta}(t) & =u(t)+\rho \eta \sin (\phi-\theta(t)),
\end{aligned}
$$

where the proportional constant, $\rho$, captures the geometry of the body of the vehicle, the location of the center of mass, and the nature of the interaction between the vehicle and the flow medium.

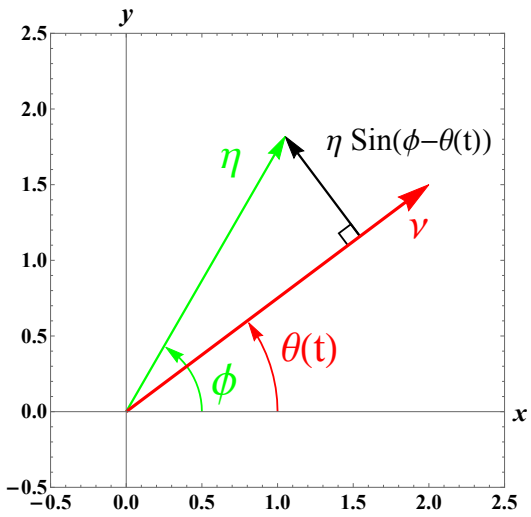

Fig. 1: Vehicle's forward speed $v$, flow speed $\eta$ and their corresponding direction angles $\theta$ and $\phi$

To clarify the significance of the developed rotational model (3), several simulations were worked out and the motion results were compared to the irrotational model in (2). For a given planar curve $(x(t), y(t))$, let $\psi=\tan ^{-1}(\dot{x}(t), \dot{y}(t))$ be the course angle representing the angle that the tangent to the curve makes with the horizontal axis.

Case 1: $u=0$ and forward velocity $v=0$. This case corresponds to placing the AUV in a constant current with a zero steering control $u=0$ and a zero forward velocity $v=0$. From Fig $2 \mathrm{a}$ it is clear that the heading angle for model (2) stays constant where as the heading angle of model (3) $\theta(t) \rightarrow \phi$ as $t \rightarrow \infty$. That is the vehicle will eventually align with the flow. However, The course angle $\psi(t)=\phi$ for both models stays constant.

Case 2: $u=0$ and forward velocity $v=1$. The resulting traversed paths are shown in Fig $2 \mathrm{~b}$. With no steering input applied to either models, $\psi(t)$ corresponding to the irrotational model (2) is constant and experiences no change, while $\psi(t)$ 


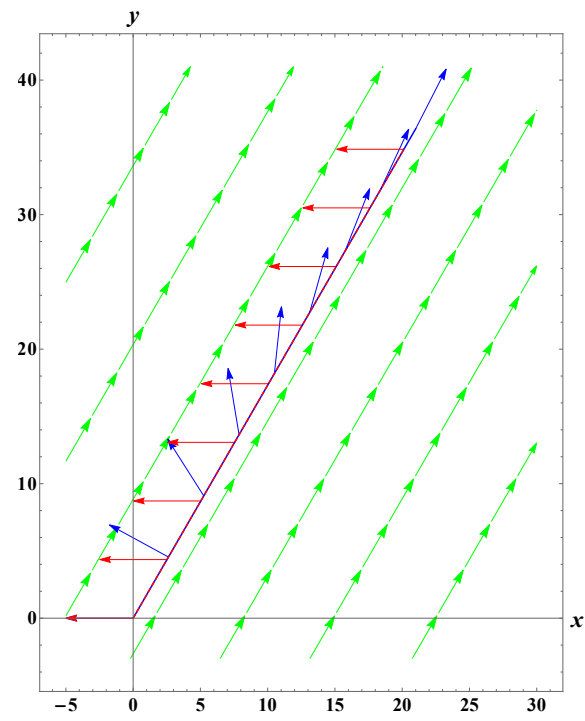

(a) Case 1 with $u=0, v=0, \theta_{\text {init }}=\pi, \rho=$ $0.1, \eta=0.35$, and $\phi=\frac{\pi}{3}$

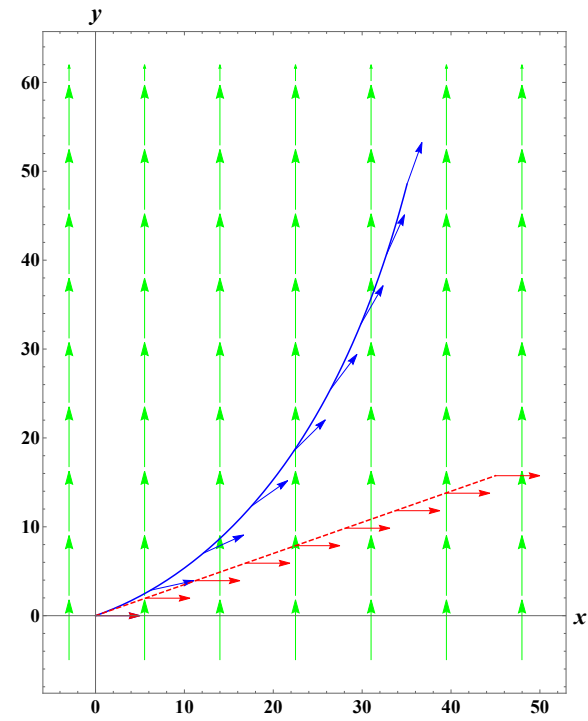

(b) Case 2 with $u=0, v=1, \theta_{\text {init }}=0, \rho=$ $0.1, \eta=0.35$, and $\phi=\frac{\pi}{2}$

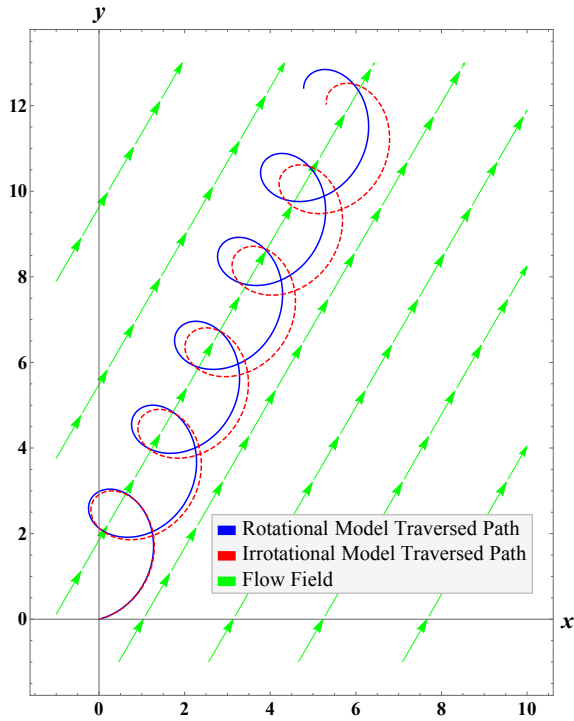

(c) Case 3 with $u=1, v=1, \theta_{\text {init }}=0, \rho=$ $0.1, \eta=0.35$, and $\phi=\frac{\pi}{3}$

Fig. 2: Rotational model versus Irrotational model for various control inputs.

corresponding to the rotational model $(3)$ is eventually aligned with $\phi$ and $\theta(t)$ as $t \rightarrow \infty$.

Case 3: $u=1$ and forward velocity $v=1$. The model in (2) traverses a trochoidal path as described in Rysdyk [20] and Techy and Woolsey [22]. However, the developed rotational model (3) shows a variation from the results described by (2) as shown in Fig 2c. It is important to stress that $\theta(t)$ is not necessarily equal to the course angle $\psi(t)$ for either models. A video depicting the simulations in Fig 2 can be found at this $\operatorname{link} 1$

From the above simulations, it is clear that the proposed model better captures the motion of a real AUV moving in a fluid medium with a constant flow.

\section{Time Optimal CONTROLS}

In order to solve the optimal path planning problem, we take recourse to optimal control theory techniques, specifically, the Pontryagin's Minimum Principle (PMP). For a minimum time problem where the end-time $t_{f}$ is free, the cost function is given by

$$
J=\int_{0}^{t_{f}} d t=t_{f}
$$

and the control Hamiltonian is given by

$$
H(\lambda, q, u, t)=g(q, u, t)+\lambda^{T} f(q, u, t),
$$

where $g$ is the integrand of the cost function $J$ and $f$ : $(q, u, t) \mapsto \dot{q}$ is the kinematic model given in (3). Using the proposed model, the Hamiltonian becomes

$$
\begin{aligned}
H(\lambda, q, u, t)= & 1+\lambda_{x}(\cos \theta(t)+\eta \cos \phi)+ \\
& \lambda_{y}(\sin \theta(t)+\eta \sin \phi)+ \\
& \lambda_{\theta}(u(t)+\rho \eta \sin (\phi-\theta(t))),
\end{aligned}
$$

\footnotetext{
1 https://www.youtube.com/watch?v=d7mpBlve2hY
}

where $\lambda=\left(\lambda_{x}, \lambda_{y}, \lambda_{\theta}\right)$ is the vector of co-state variables. These variables are governed by the costate equations given by

$$
\begin{aligned}
\dot{\lambda}_{x}= & -\frac{\partial H}{\partial x}=0, \\
\dot{\lambda}_{y}= & -\frac{\partial H}{\partial y}=0, \\
\dot{\lambda}_{\theta}= & -\frac{\partial H}{\partial \theta} \\
= & \left(\lambda_{x}-\lambda_{\theta} \rho \eta \cos \phi\right) \sin \theta(t) \\
& +\left(\lambda_{\theta} \rho \eta \sin \phi-\lambda_{y}\right) \cos \theta(t) .
\end{aligned}
$$

According to Pontryagin's Minimum Principle the optimal control, $u^{*}$, is the one that minimizes the Hamiltonian such that, $H\left(\lambda^{*}, q^{*}, u^{*}, t\right) \leqslant H\left(\lambda^{*}, q^{*}, u, t\right)$. Also for a free endtime problem, using Kirk [14], the boundary condition is given by $H\left(\lambda^{*}\left(t_{f}\right), q^{*}\left(t_{f}\right), u^{*}\left(t_{f}\right), t_{f}\right)=0$. Additionally, since the Hamiltonian is not explicit in time we arrive at

$$
H\left(\lambda^{*}(t), q^{*}(t), u^{*}(t)\right)=0
$$

The Hamioltonian in 6 is linear in the control,then minimizing the Hamiltonian with respect to $u(t)$ is equivalent to minimizing $\lambda_{\theta}(t) u(t)$. Thus, the optimal control, $u^{*}(t)$, is a function of the sign of $\lambda_{\theta}(t)$ which is also known as the switching function. To find the optimal controls for the proposed model the following cases are considered.

A. Case where $\lambda_{\theta}=0$ :

Using (10) the Hamiltonian becomes $H=1+\lambda_{x}(\cos \theta(t)+$ $\eta \cos \phi)+\lambda_{y}(\sin \theta(t)+\eta \sin \phi)=0$. Even though $u(t)$ doesn't appear explicitly, it is still possible to deduce important results regarding the control input. From the costate equations, (7) and (8), and from the assumption of constant flow, the 
following variables, $\lambda_{x}, \lambda_{y}, \eta$, and $\phi$, are constants. Thus, the Hamiltonian becomes

$$
\lambda_{x} \cos \theta(t)+\lambda_{y} \sin \theta(t)=\text { constant },
$$

which implies that $\theta(t)=$ constant $(\bmod 2 \pi)$. Thus, the heading angle, $\psi(t)$, is constant which yields a straight line path. Additionally, since $\theta(t)$ is constant, using the third equation in (3), one can solve for the optimal control, which will be referred to as a singular control, to get

$$
u^{*}(t)=-\rho \eta \sin (\phi-\theta(t)) .
$$

\section{B. Case where $\lambda_{\theta} \neq 0$ :}

The optimal control input, $u^{*}(t)$, that minimizes the Hamiltonian whenever $\lambda_{\theta} \neq 0$ is given by,

$$
u^{*}=-\operatorname{sign}\left(\lambda_{\theta}\right) u_{\max },
$$

which represents a maximum turning control either to the left or the right, also known as a maximum effort or a bang control given by

$$
u *(t)= \pm 1 .
$$

Hence, Pontryagin's Minimum Principle reduces the space of candidate minimum time paths to those traversed by the model under the two optimal controls, the singular controls in (12) resulting in straight line paths, and the maximum effort controls in (14) resulting in stretched-like trochoids.

\section{Structure of Motion Primitives}

Having reduced the space of candidate minimum time paths to concatenations of straight lines and turning segments corresponding to maximum effort control inputs, in this section, these motion primitives are characterized and their structure is analyzed to be used in the path synthesis problem. First some terminology is introduced.

- $C_{a}$ : A curve traversed by the vehicle when taking a maximum turn rate either to the left or the the right so $C$ could take the values of $L$ or $R$ and $a$ is either $i$ indicating initial or $f$ indicating final.

- $t_{i L}$ : time spent traversing a maximum left turning segment starting from the initial configuration $q_{i}=\left\{x_{i}, y_{i}, \theta_{i}\right\}$. Similarly $t_{i R}$ is defined to correspond to a right turn.

- $t_{f L}$ : time spent traversing a maximum left turning segment ending at the final configuration $q_{f}=\left\{x_{f}, y_{f}, \theta_{f}\right\}$. Similarly $t_{f R}$ is defined to correspond to a right turn.

- $q_{i L}(t)$ : corresponds to a point starting at an initial configuration and traversing a maximum left turn for a duration $t$. Similarly $q_{i R}(t)$ is defined for a right turn.

- $S_{C_{i}, C_{f}}$ : straight line segment that is tangent to both the initial turning segment $C_{i}$ and final turning segment $C_{f}$.

- $V_{C_{i}, C_{f}}$ : velocity of the particle while traveling along the straight segment tangent to both $C_{i}$ and $C_{f}$.

\section{A. Maximum Turning Rate Segments}

Pontryagin's Minimum Principle restricts the segments of any optimal path for the rotational model $(3)$ into maximum turning rate segments and straight line segments. Some important implications of such results are presented below.

Using Mathematica, an analytical solution for the non-linear differential equation describing $\theta(t)$ in the rotational model in $\sqrt{3}$ is given by

$$
\theta(t)=\phi+2 \tan ^{-1}\left(\frac{\left.\eta \rho+\sqrt{u^{2}-\eta^{2} \rho^{2}} \tan \beta\right)}{u}\right)
$$

where $\beta$ is

$$
\beta=\frac{1}{2} t \sqrt{u^{2}-\eta^{2} \rho^{2}}+\tan ^{-1}\left(\frac{u \tan \left(\frac{\theta_{0}-\phi}{2}\right)-\eta \rho}{\sqrt{u^{2}-\eta^{2} \rho^{2}}}\right) .
$$

Inspecting the above solution, specifically the term associated with time, $t$, it is clear that $\theta(t)$ is periodic with a period

$$
P=\frac{2 \pi}{\sqrt{u^{2}-\rho^{2} \eta^{2}}}
$$

Under constant flow conditions and a constant bang control, such as $u(t)=1$, the net displacement vector generated by a full turning period of $\theta(t)$, denoted by $D_{2 \pi}$, is unique and independent of the initial configuration. That is, starting from any initial fixed position $\{x(0), y(0)\}$, then taking a maximum rate left turn for a period $P$, the final position $\{x(P), y(P)\}$ is the same independent of the initial heading $\theta(0)$ as shown in Fig. 3. The arrows in Fig. 3 depict the initial and final headings corresponding to each maximum left turn curve. Similarly $u(t)=-1$ defines a unique net displacement vector for a full period right turn. Hence, the shapes of bang path are periodic. This can be verified by integrating the first two equations in (3) over a full period of $\theta(t)$.

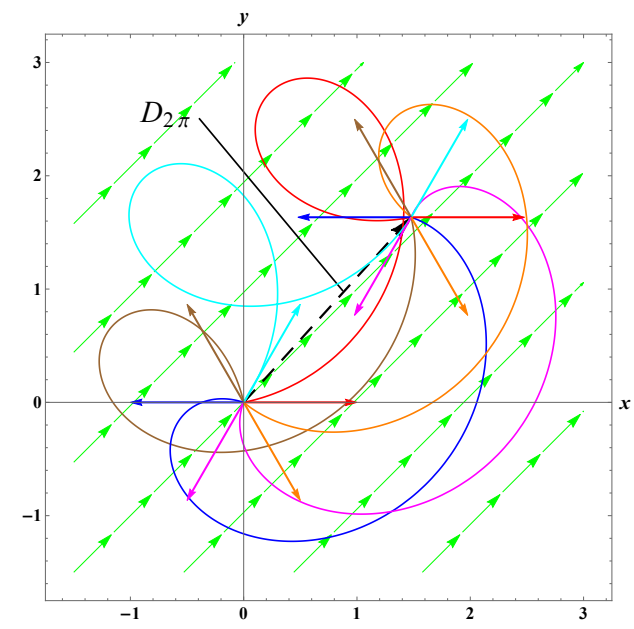

Fig. 3: Full Period Maximum Effort Left Turns starting form different initial headings under fixed flow conditions 


\section{B. Straight Segments}

For the case where $\theta$ is constant, and given that $u \in[-1,1]$, it is clear from equation (11) that to achieve singular segments, one must assume that $|\rho \eta|<1$. Unlike prior models where the control $u$ takes only the discrete values 1,0 , or -1 , in the proposed model, for singular segments, $u$ could take any feasible value to ensure that $\theta$ is constant. Thus, for the rest of the paper, it is assumed that flow parameters are constrained by $|\rho \eta|<1$.

\section{Optimal Path Synthesis}

Having proved that any time optimal path must consist of either singular straight segments or bang maximum turning rate segments, the number and order of concatenations of such segments, that is, the path synthesis problem, is addressed next.

\section{A. Concatenations of Possible Path Segments}

The first two equations of the proposed rotational model (3) describing $\dot{x}(t)$ and $\dot{y}(t)$ can be written in the form of the model introduced by Dolinskaya and Maggiar [10] which is given by

$$
\begin{aligned}
& \dot{x}(t)=V(\theta) \cos \theta(t), \\
& \dot{y}(t)=V(\theta) \sin \theta(t), \\
& \dot{\theta}(t)=\frac{V(\theta)}{R(\theta)} u(t),
\end{aligned}
$$

where $V(\theta)$ is the vehicle's net speed in polar coordinates and $R(\theta)$ is the minimum turning radius of the vehicle also in polar coordinates. Recall that the two components, $x(t)$ and $y(t)$, describe the path traversed by the vehicle. Thus, results obtained by Dolinskaya and Maggiar [10] on path segment concatenations are applicable to the proposed rotational model in (3). Hence, as indicated in [10], the number of segment concatenations and the structure of the path are a function of the convexity of $V(\theta)$, and can be summarized as follows:

1) Non-Convex Speed Polar Plots: if a path intersects a non-convex portion of the speed polar plot, the optimal path is comprised of five segments having the following specific order CSCSC.

2) Convex Speed Polar Plots: if a path intersects a convex portion of the speed polar plot, then the optimal path is comprised of at most three sections which have the following order $C S C$ or $C C C$.

\section{B. Convexity Condition}

Restricting the path synthesis problem in this paper to either CSC to CCC, another constraint is imposed on the flow parameters in such a way to ensure that the speed polar plot is convex everywhere.

The speed in polar coordinates for the proposed model is given by

$$
V(\theta)=\sqrt{\dot{x}^{2}+\dot{y}^{2}}=\sqrt{\eta^{2}+2 \eta \cos (\theta-\phi)+1} .
$$

Note that, the net speed polar plot in the absence of any flow, i.e. $\eta=0$, is simply the unit circle depicted as a dashed circle in Fig 4 The effect of constant flow will deform this unit circle, where the amount and location of deformation, as expected, depends on the flow parameters, $\eta$ and $\phi$.

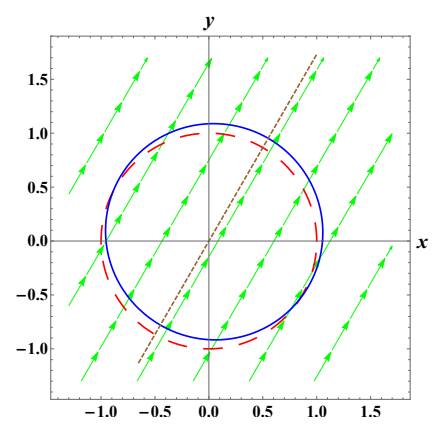

(a) $\eta=0.1$ and $\phi=\frac{\pi}{3}$

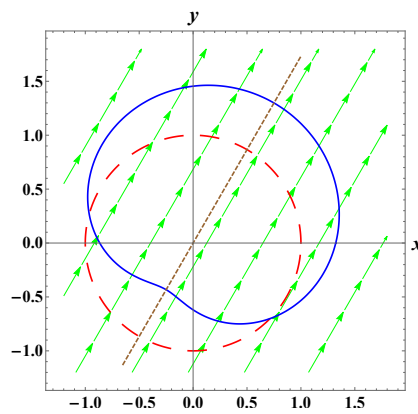

(c) $\eta=0.5$ and $\phi=\frac{\pi}{3}$

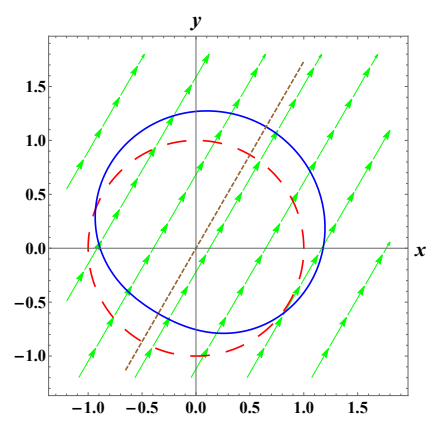

(b) $\eta=0.3$ and $\phi=\frac{\pi}{3}$

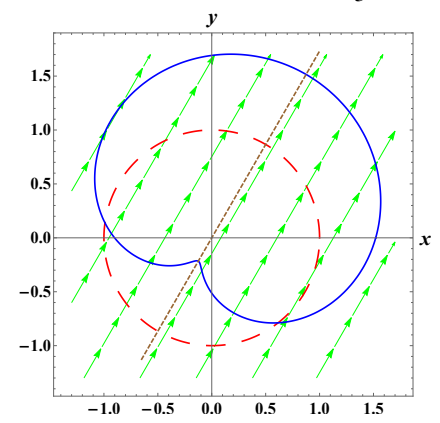

(d) $\eta=0.75$ and $\phi=\frac{\pi}{3}$
Fig. 4: Polar plots of the maximum net speed for different $\eta$

Studying the effect of the constant flow on the speed polar plot, as shown in Fig. 4, it is clear that the maximum deformation to the polar plot will occur along the direction of the flow, that is, at $\theta=\phi+\pi$. Let $\alpha=\theta-\phi$, then the curvature in polar coordinates is given by

$$
\kappa=\frac{V^{2}+2\left(\frac{\partial V}{\partial \alpha}\right)^{2}-V \frac{\partial^{2} V}{\partial \alpha \partial \alpha}}{\left(V^{2}+\left(\frac{\partial V}{\partial \alpha}\right)^{2}\right)^{3 / 2}} .
$$

Setting the numerator of the curvature, $\kappa$, in 20 to zero, will determine the flow speed, $\eta$, at which the deformation in the speed polar plot starts to form a non-convex region in the plot. The numerator in terms of $\alpha$ is given by

$$
\begin{aligned}
10\left(\eta^{3}+\eta\right) \cos (\alpha) & +3 \eta^{2} \cos (2 \alpha) \\
+ & 2 \eta^{4}+13 \eta^{2}+2=0 .
\end{aligned}
$$

Setting $\alpha=\pi$, then the only feasible root of the equation turns out to be $\eta=\frac{1}{2}(3-\sqrt{5})$ which is the maximum flow velocity for which the entire speed polar plot will remain convex.

Having characterized the individual segments and the concatenations of such segments, and by setting $\eta \leq 0.382$, the model is then restricted to the case where the speed polar 


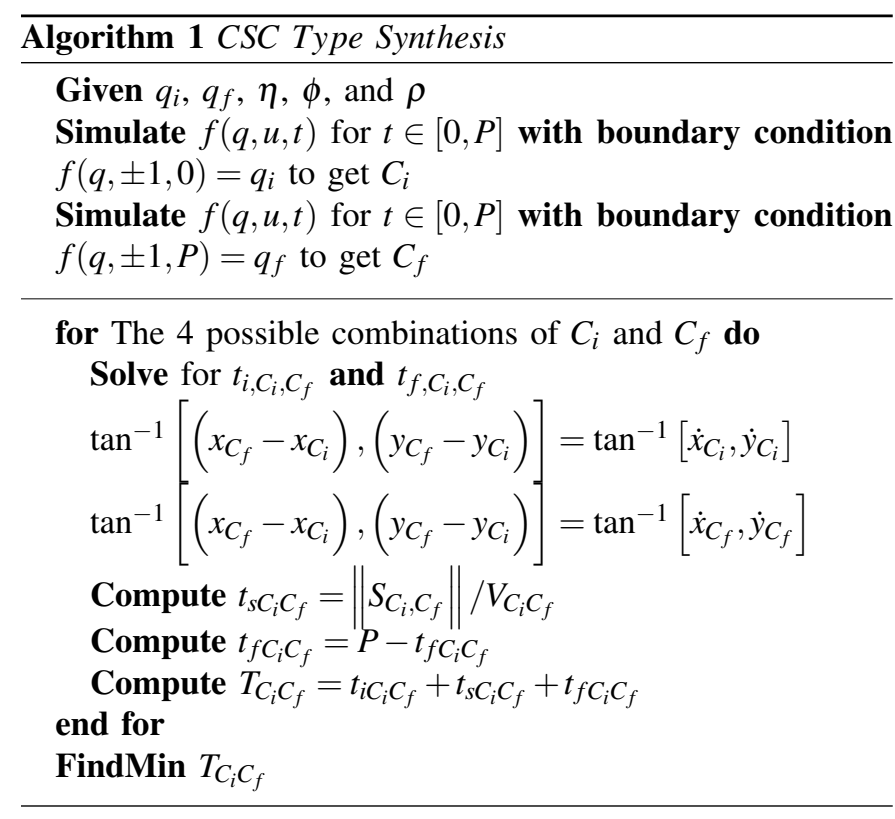

plot is convex. This restriction guarantees that an optimal path takes either the form $C S C$ or $C C C$. This reduces the space of possible optimal paths to a maximum of six types: $L S L, R S R$, $R S L, L S R, L R L$, and $R L R$.

\section{CSC Type Path Synthesis}

There exists four candidate minimum time paths of type $C S C$ between an initial configuration and a final one, namely $L S L, L S R, R S R$, and $R S L$. For a given pair of initial and final configurations where the Euclidean distance between $q_{i}$ and $q_{f}$ is greater than 4||$D_{2 \pi}||$ it is guaranteed that the solution will be of $C S C$ type.

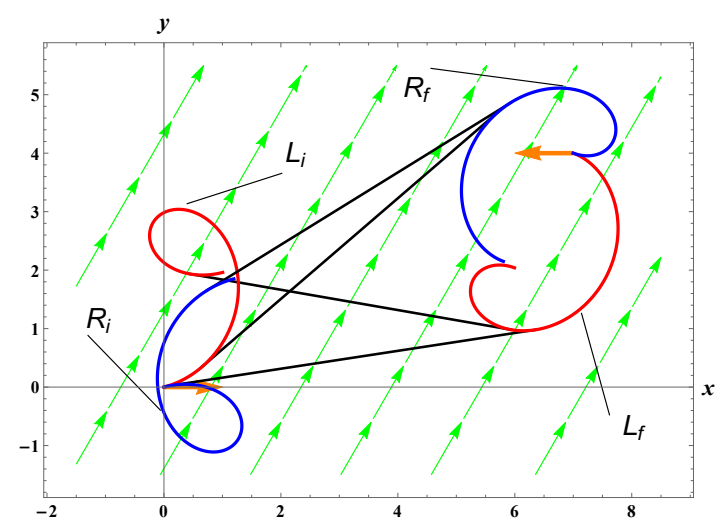

Fig. 5: $C S C$ path synthesis for $q_{f}=\{8,4, \pi\}, \eta=0.35$, and $\phi=\frac{\pi}{3}$

Algorithm 1 constructs all four possible CSC paths then finds the path corresponding to the minimum travel time. In order to accomplish this, the algorithm first constructs the initial maximum turning rate curves starting from $\{x(0), y(0), \theta(0)\}=q_{i}$ to the right and left respectively labeled as $R_{i}$ and $L_{i}$. Next, maximum turning rate segments

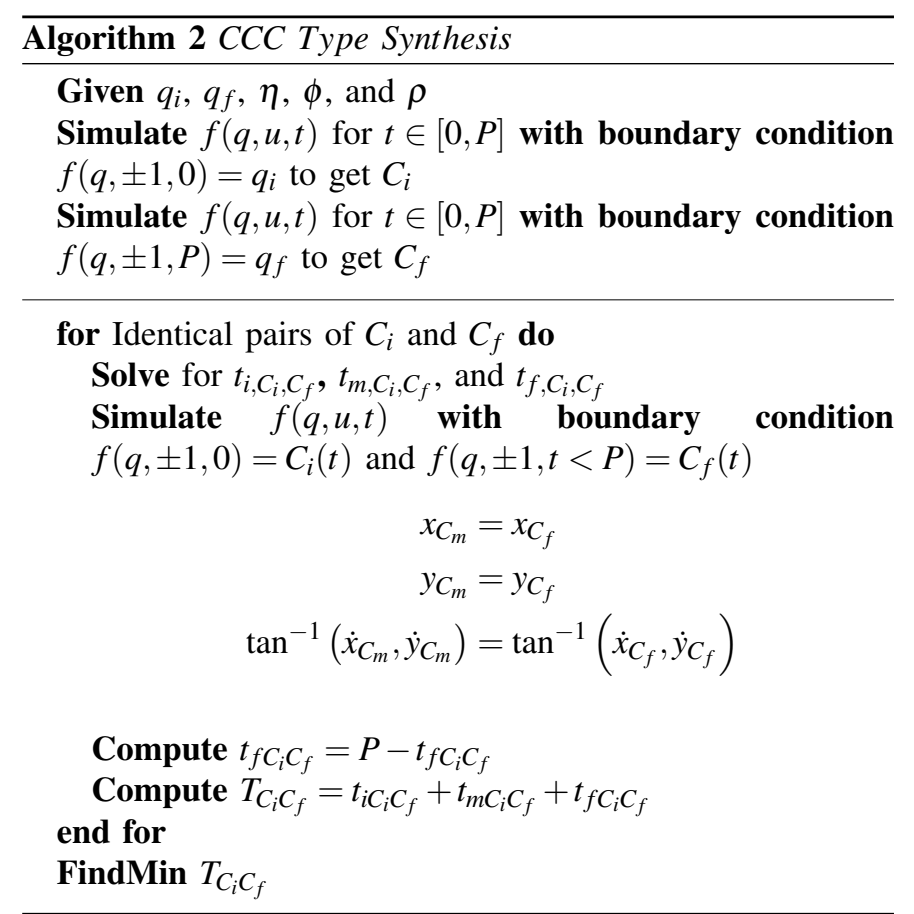

are constructed to end at $\{x(P), y(P), \theta(P)\}=q_{f}$ turning to the right and left respectively labeled as $R_{f}$ and $L_{f}$. Then, the algorithm seeks a line segment connecting a point on $C_{i}$ to a point on $C_{f}$ such that the line segment is tangent to both curves. Finally, the algorithm computes the total time of travel along each of the four possible paths and finds the path corresponding to the minimum travel time. Fig 5 shows the initial and final maximum rate turning curves for a full period $P$ along with the corresponding tangents.

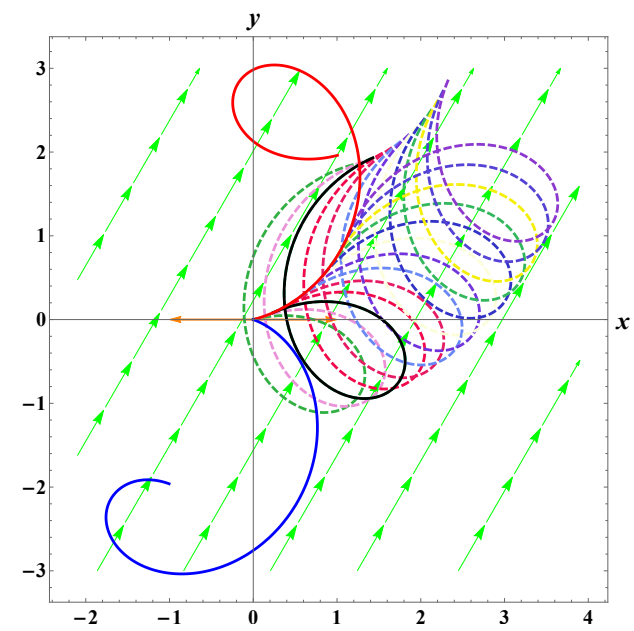

Fig. 6: $L_{i}$ and $L_{f}$ with $q_{i}=(0,0,0), q_{f}=(0,0, \pi)$ and multiple right turning segments starting on $L_{i}$

\section{CCC Type Synthesis}

For cases where the Euclidean distance between $q_{i}$ and $q_{f}$ is less than 4||$D_{2 \pi} \|$, a mid-curve or type $C_{m}$ could possibly be 


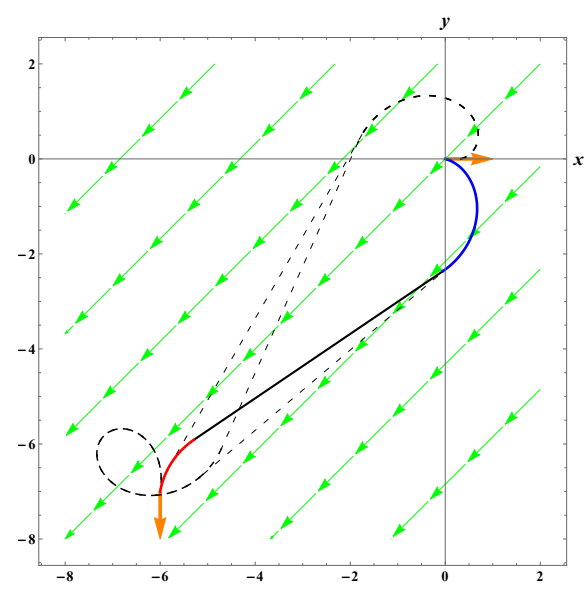

(a) Time Optimal Path for $\eta=0.3, \phi=\frac{-3 \pi}{4}$, $q_{f}=\left\{-6,-7, \frac{-\pi}{2}\right\}$ is $R S L$ with $t=8.48 s$

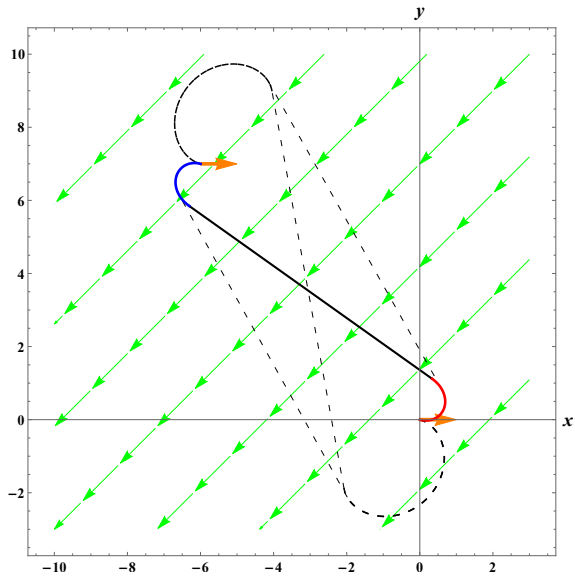

(b) Time Optimal Path for $\eta=0.3, \phi=\frac{-3 \pi}{4}$, $q_{f}=\{-6,7,0\}$ is $L S R$ with $t=12.56 s$

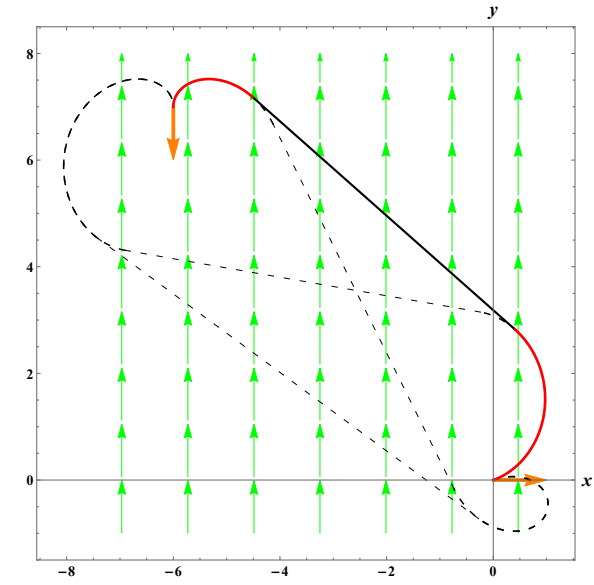

(c) Time Optimal Path for $\eta=0.35, \phi=\frac{\pi}{2}$, $q_{f}=\left\{-6,7, \frac{-\pi}{2}\right\}$ is $L S L$ with $t=10.23 \mathrm{~s}$

Fig. 7: Time optimal paths for cases where only paths of type CSC are possible

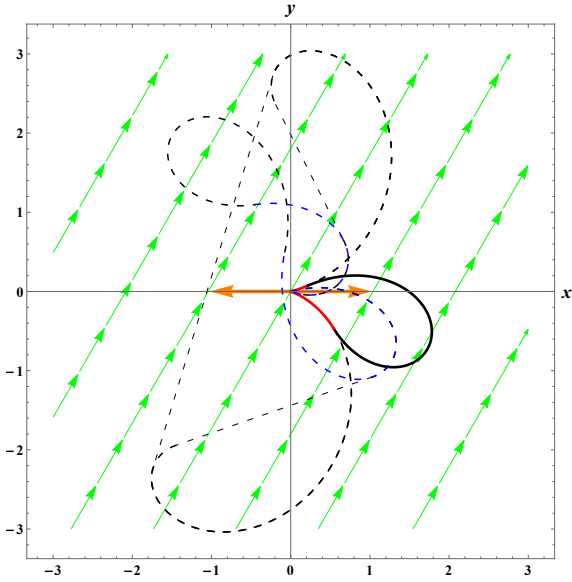

(a) Time Optimal Path for $\eta=0.35, \phi=\frac{\pi}{3}$, $q_{f}=\{0,0, \pi\}$ is $L R L$ with $t=4.98 s$

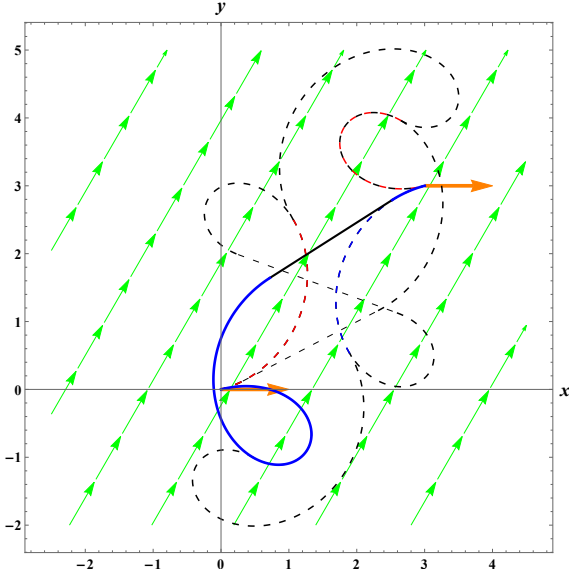

(b) Time Optimal Path for $\eta=0.35, \phi=\frac{\pi}{3}$, $q_{f}=\{3,3,0\}$ is $R S R$ with $t=7.93 s$

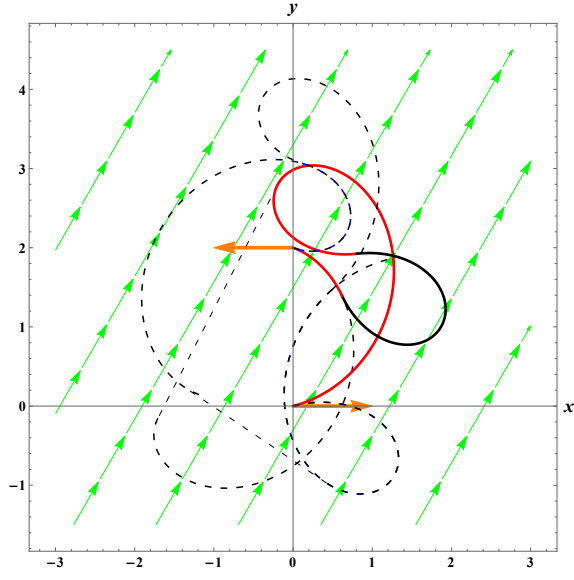

(c) Time Optimal Path for $\eta=0.35, \phi=\frac{\pi}{3}$, $q_{f}=\{0,2, \pi\}$ is $L R L$ with $t=10.8 s$

Fig. 8: Cases where both types $C S C$ and $C C C$ are time optimal candidates

tangent to both $C_{i}$ and $C_{f}$. Fig 6 shows an example where multiple right turning middle curves, depicted by dashed curves, starting initially from different points along the initial left turning segment intersect with the final turning segment. Also note that one of the mid-curves, depcited as a solid curve, is also tangent to $L_{f}$. For such case, a $C C C$ type curve is a possible minimum time candidate. Algorithm 2 solves for $C C C$ candidates only. Possible path types are either $R L R$ or $L R L$.

Algorithm 2 first constructs both $L$ and $R$ initial and final turning segments. After that a turning segment with opposite direction to that of the initial turning segment and having initial conditions be any point belonging to the initial turning segment is constructed. Then for each pair of same direction initial and final turning segments $C_{i}$ and $C_{f}$, an opposite direction turning segment $C_{m}$ is solved for numerically. $C_{m}$ starts from a point and a heading on $C_{i}$ and is tangent to $C_{f}$. Finally the total travel time corresponding to each candidate path is computed and and the path corresponding to the minimum travel time is found. However, for the case where CCC paths are possible candidates, CSC paths are also still valid candidates for minimum time paths. Thus, an algorithm combining both algorithms 1 and 2 computes the minimum time path in such a case.

\section{Simulations}

Some results of the algorithms introduced earlier are presented in this section. Without loss of generality, the initial configuration is assumed to be $q_{i}=\{0,0,0\}$ for all cases. Also, $\rho$ is assumed to equal 0.1 for all cases presented in Fig. 7 and Fig. 8 The flow velocity $\eta$ is kept close to the limit, that is 0.38 , to ensure that the speed polar plot is convex.

Fig. 7 depicts cases where all possible path concatenations are of the type CSC. Three different cases are presented where the final configuration, $q_{f}$, as well as the flow parameters, $\phi$ 


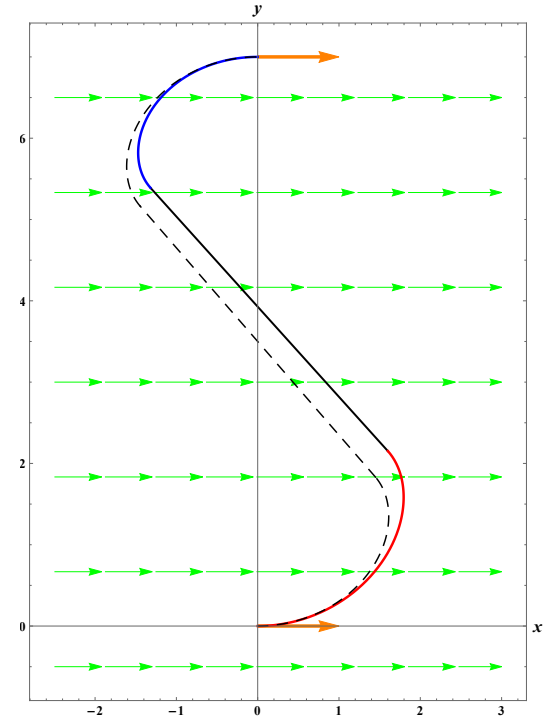

(a) Time Optimal Path for $\eta=0.35, \phi=$ $0, q_{f}=\{0,7,0\}$ is $L S R$ with $t=11.12 s$

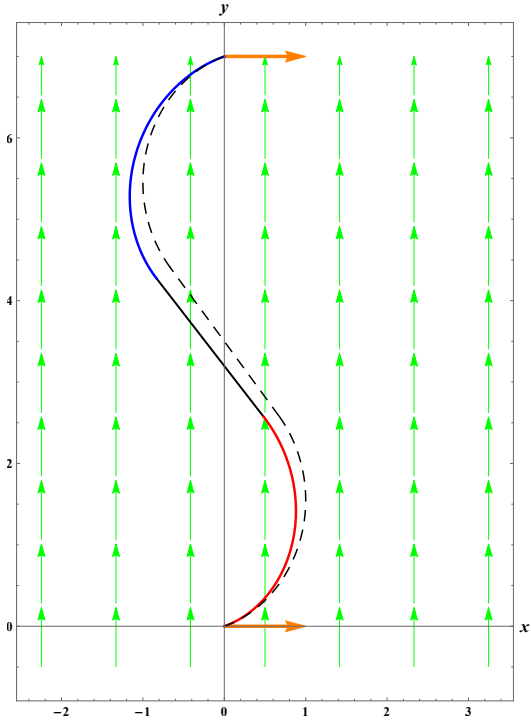

(b) Time Optimal Path for $\eta=0.35, \phi=$ $\frac{\pi}{2}, q_{f}=\{0,7,0\}$ is $L S R$ with $t=6.68 s$

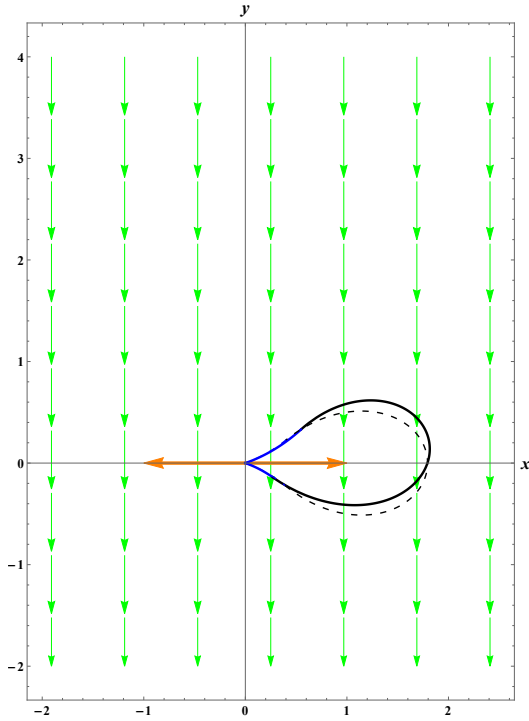

(c) Time Optimal Path for $\eta=0.35, \phi=$ $\frac{-\pi}{2}, q_{f}=\{0,0, \pi\}$ is $L R L$ with $t=5.83 \mathrm{~s}$

Fig. 9: Optimal paths for the Rotational model versus the Irrotational model

and $\eta$, are changed. For each case, the four possible path concatenations $L S L, R S R, L S R$ and $R S L$ are always guaranteed to exist as depicted by the dashed curves. The path corresponding to the minimum time of travel of the vehicle is depicted using solid curves. Note that, the turning radius and length of various the turning segments depend on the flow parameters as well as the heading at which the vehicle starts to take a turn.

Several cases where the paths are potentially of the type $C C C$ are as shown in Fig. 8 Not that, for such cases, considering the initial and final turning segments, one has to check, if these turning segments intersect or not. If the segments intersect, then the potential candidate curve concatenation is of $C C C$ type, whereas if the turning segment do not intersect, the potential candidate curve concatenation is of CSC type. Referring to Fig. 8b, the candidate curve are $L R L, R L R$, as well as $L S L, R S R$, and $L S R$. In this particular case, the shortest path is the $R S R$. The proposed algorithm attempts to solve for all possible paths, and identified the time optimal one whether it is a $C C C$ or a $C S C$ type. As for Fig. $8 \mathrm{a}$ and Fig $8 \mathrm{c}$, the time optimal path for both is of type $L R L$.

Fig. 9] compares the optimal paths for both the rotational and irrotational models where $\rho$ is assumed to equal 0.5 . The dashed paths represent the optimal path corresponding to the irrotational model whereas the solid paths correspond to the rotational model. Accounting for a rotational effect in the model shows that it is possible to traverse turning curves with a smaller turning radius compared to the irrotational model as shown in the final left turn of Fig. 9a. On the other hand, for different scenarios such as the initial left turn of Fig. 9a or the final right turn of the path in Fig. $9 b$ it is only possible to follow the corresponding curves with radii greater than those of the turns corresponding to the irrotational model.

In Fig. 9c the maneuver of parking in the same position but with an opposite direction is considered, that is, going from $q_{i}=(0,0,0)$ to $q_{f}=(0,0, \pi)$. For this case, the optimal path is $L R L$ for both models. However, note that for the irrotational model, the solution path is symmetric about the $x$-axis. This, does not represent accurately the effect of a flow on a vehicle. As for the rotational model, the effect of the flow breaks the symmetry of the curve and yields a more reasonable solution path. A video depicting the simulations in Fig 9 can be found at this link 2

\section{CONCLUSION}

This paper presented a modified model that incorporates not only a transnational effect due to a flow, but also accounts for the effect induced on the turning rate of an AUV traveling through a constant flow. Then using Pontryagin's Minimum Principle, motion primitives which are the building blocks for an optimal path were obtained. The structure of the obtained path segments were characterized and analyzed in order to propose robust path planning algorithm.

In the future work, the proposed model will be validated against an actual vehicle moving withing a constant flow. Also, the proposed path planning algorithms need to be modified to accommodate non-convex speed polar plots. Finally, other cost function should be considered such as minimum control effort or minimum energy.

\section{ACKNOWLEDGMENTS}

This work was supported by the University Research Board (URB) at the American University of Beirut (AUB) and the National Council for Scientific Research (CNRS), Lebanon.

\footnotetext{
2 https://www.youtube.com/watch?v=7VkjlAKsmpk
} 


\section{REFERENCES}

[1] Efstathios Bakolas and Panagiotis Tsiotras. Time-optimal synthesis for the Zermelo-Markov-Dubins problem: the constant wind case. In American Control Conference (ACC), pages 6163-6168, 2010.

[2] Efstathios Bakolas and Panagiotis Tsiotras. Optimal synthesis of the asymmetric sinistral/dextral MarkovDubins problem Journal of optimization theory and applications, 150(2):233-250, 2011.

[3] Devin J Balkcom and Matthew T Mason. Time optimal trajectories for bounded velocity differential drive vehicles The International Journal of Robotics Research, 21 (3):199-217, 2002.

[4] Devin J Balkcom, Paritosh A Kavathekar, and Matthew T Mason. Time-optimal trajectories for an omni-directional vehicle The International Journal of Robotics Research, 25(10):985-999, 2006.

[5] Jean-Daniel Boissonnat, André Cérézo, and Juliette Leblond. Shortest paths of bounded curvature in the plane. Springer, 1993.

[6] Xuân-Nam Bui, Jean-Daniel Boissonnat, Philippe Soueres, and Jean-Paul Laumond. Shortest path synthesis for Dubins non-holonomic robot In Robotics and Automation, 1994. Proceedings., 1994 IEEE International Conference on, pages 2-7. IEEE, 1994.

[7] Alan J Chang, Marcus Brazil, J Hyam Rubinstein, and Doreen A Thomas. Curvature-constrained directionalcost paths in the plane. Journal of Global Optimization, 53(4):663-681, 2012.

[8] Hamidreza Chitsaz and Steven M LaValle. Time-optimal paths for a Dubins airplane. In Decision and Control, 2007 46th IEEE Conference on, pages 2379-2384. IEEE, 2007.

[9] Heejun Choi. Time-Optimal Paths for a Dubins Car and Dubins Airplane with a Unidirectional Turning Constraint PhD thesis, The University of Michigan, 2014.

[10] Irina S Dolinskaya and Alvaro Maggiar. Time-optimal trajectories with bounded curvature in anisotropic media The International Journal of Robotics Research, 31(14): 1761-1793, 2012.

[11] Irina S Dolinskaya, M Kotinis, MG Parsons, and
RL Smith. Optimal short-range routing of vessels in a seaway. Journal of Ship Research, 53(3):121-129, 2009.

[12] Lester E Dubins. On curves of minimal length with a constraint on average curvature, and with prescribed initial and terminal positions and tangents American Journal of mathematics, pages 497-516, 1957.

[13] Andrei A Furtuna and Devin J Balkcom. Generalizing Dubins curves: Minimum-time sequences of body-fixed rotations and translations in the plane. The International Journal of Robotics Research, 2010.

[14] Donald E Kirk. Optimal control theory: an introduction Courier Corporation, 2012.

[15] Andrey Andreyevich Markov. Some examples of the solution of a special kind of problem on greatest and least quantities. Soobshch. Karkovsk. Mat. Obshch, 1: 250-276, 1887.

[16] Timothy G McGee and J Karl Hedrick. Optimal path planning with a kinematic airplane model Journal of guidance, control, and dynamics, 30(2):629-633, 2007.

[17] Rachelle L McNeely, Ram V Iyer, and Phillip R Chandler. Tour planning for an unmanned air vehicle under wind conditions Journal of Guidance, Control, and Dynamics, 30(5):1299-1306, 2007.

[18] Lev Semenovich Pontryagin. Mathematical theory of optimal processes. CRC Press, 1987.

[19] James Reeds and Lawrence Shepp. Optimal paths for a car that goes both forwards and backwards Pacific journal of mathematics, 145(2):367-393, 1990.

[20] Rolf Rysdyk. Course and heading changes in significant wind Journal of guidance, control, and dynamics, 30 (4):1168-1171, 2007.

[21] Héctor J Sussmann and Guoqing Tang. Shortest paths for the Reeds-Shepp car: a worked out example of the use of geometric techniques in nonlinear optimal control. Rutgers Center for Systems and Control Technical Report, 10:1-71, 1991.

[22] Laszlo Techy and Craig A Woolsey. Minimum-time path planning for unmanned aerial vehicles in steady uniform winds Journal of guidance, control, and dynamics, 32 (6):1736-1746, 2009. 